\title{
Genome sequence of Apostasia ramifera provides insights into the adaptive evolution in orchids
}

Weixiong Zhang ${ }^{1 \dagger}$, Guoqiang Zhang ${ }^{2,3,4+}$, Peng Zeng ${ }^{1 \dagger}$, Yongqiang Zhang ${ }^{2,3,4,5}$, Hao Hu' ${ }^{1}$ Zhongjian Liu ${ }^{5}$ and Jing $\mathrm{Cai}^{\mathrm{6}^{*}}$

\begin{abstract}
Background: The Orchidaceae family is one of the most diverse among flowering plants and serves as an important research model for plant evolution, especially "evo-devo" study on floral organs. Recently, sequencing of several orchid genomes has greatly improved our understanding of the genetic basis of orchid biology. To date, however, most sequenced genomes are from the Epidendroideae subfamily. To better elucidate orchid evolution, greater attention should be paid to other orchid lineages, especially basal lineages such as Apostasioideae.

Results: Here, we present a genome sequence of Apostasia ramifera, a terrestrial orchid species from the Apostasioideae subfamily. The genomes of $A$. ramifera and other orchids were compared to explore the genetic basis underlying orchid species richness. Genome-based population dynamics revealed a continuous decrease in population size over the last 100000 years in all studied orchids, although the epiphytic orchids generally showed larger effective population size than the terrestrial orchids over most of that period. We also found more genes of the terpene synthase gene family, resistant gene family, and LOX1/LOX5 homologs in the epiphytic orchids.
\end{abstract}

Conclusions: This study provides new insights into the adaptive evolution of orchids. The A. ramifera genome sequence reported here should be a helpful resource for future research on orchid biology.

Keywords: Orchidaceae, Apostasia ramifera, Comparative genomics, Adaptive evolution

\section{Background}

The Orchidaceae family is one of the largest among flowering plants, with many species exhibiting great ornamental value due to their colorful and distinctive flowers. At present, there are more than 28000 orchid species assigned to 763 genera [1]. According to their phylogeny, orchids can be divided into five subfamilies,

\footnotetext{
* Correspondence: jingcai@nwpu.edu.cn

Weixiong Zhang, Guogiang Zhang, and Peng Zeng are co-first authors

tWeixiong Zhang, Guoqiang Zhang and Peng Zeng contributed equally to this work.

${ }^{6}$ School of Ecology and Environment, Northwestern Polytechnical University, 710129 Xi'an, China

Full list of author information is available at the end of the article
}

i.e., Apostasioideae, Vanilloideae, Cypripedioideae, Epidendroideae, and Orchidoideae. It has been proposed that whole-genome duplication occurred in the ancestor of all orchid species, which contributed to their survival under significant climatic change [2,3]. Orchids are a diverse and widespread family of flowering plants. Notably, several orchid species with specialized floral structures, such as labella and gynostemia, appear to have coevolved with animal pollinators to facilitate reproductive success. In addition to their role in research on evolution and pollination biology, orchids are invaluable to the horticultural industry due to their elegant and distinctive flowers [4]. 
The genome sequences of several orchid species have been published recently, thereby greatly improving our understanding of orchid biology and evolution. The first reported orchid genome (Phalaenopsis equestris) showed evidence of an ancient whole-genome duplication event in the orchid lineage and revealed that expansion of $M A D S$-box genes may be related to the diverse morphology of orchid flowers [2]. The subsequent publication of other orchid genome sequences, such as that of Dendrobium officinale, Dendrobium catenatum, Phalaenopsis aphrodite, Apostasia shenzhenica, and Vanilla planifolia, has provided data for further investigations on the genetic mechanisms underlying orchid species richness $[3,5-8]$.

The Apostasioideae subfamily consists of terrestrial orchid species [9]. Species within Apostasioideae exhibit various primitive traits, such as radially symmetrical flowers and no labella, supporting the placement of this subfamily as a sister clade to all other orchids [10]. These primitive features are considered ancient characteristics of the orchid lineage [10]. Thus, Apostasioideae species can serve as an important outgroup for evolutionary study of all other orchid subfamilies. Recently, Zhang et al. [3] published the A. shenzhenica genome and identified an orchid-specific whole-genome duplication event as well as changes in the MADS-box gene family associated with different orchid characteristics. This is the first (and only) genome reported for the Apostasioideae subfamily, with most currently published genomes belonging to the Epidendroideae subfamily. Obtaining genomes for other orchid lineages, especially basal lineages, will greatly facilitate our understanding of orchid evolution. Here, we performed de novo assembly and analysis of the Apostasia ramifera genome sequence, the second Apostasia genome after $A$. shenzhenica. Comparative genomics were carried out with six other published orchid genomes to provide insight into orchid evolution.

\section{Results}

\section{Genome sequencing and assembly}

The genomic DNA of $A$. ramifera was sequenced using the Illumina Hiseq 2000 platform. Sequencing of five libraries with different insert sizes ranging from 250 to 5 000 bp generated more than $57 \mathrm{~Gb}$ of clean data, accounting for 156X of the genome sequence (Additional file 1, Table S1). Based on the clean reads, we generated a $365.59-\mathrm{Mb}$ long assembly with a scaffold N50 of $287.45 \mathrm{~kb}$ (Table 1 and Additional file 1, Table S2). To assess the quality of the final assembly, clean reads were mapped to the genome sequence, resulting in a mapping ratio of $99.7 \%$. The completeness of the gene regions in the assembly was examined by BUSCO (Benchmarking Universal Single-Copy Orthologs) assessment [11]. In total, $94.9 \%$ (1 304/1 375) of the universal single-copy
Table 1 Statistics related to A. ramifera genome assembly

\begin{tabular}{ll}
\hline Feature & Summary \\
\hline Genome Size & $365588417 \mathrm{bp}$ \\
Scaffold N50 & $287449 \mathrm{bp}$ \\
Contig N50 & $30765 \mathrm{bp}$ \\
Longest Scaffold & $1388560 \mathrm{bp}$ \\
GC Rate & $33.38 \%$ \\
Repeat Content & $44.99 \%$ \\
BUSCO Assessment & $94.9 \%$ \\
Gene Number & 22841 \\
\hline
\end{tabular}

orthologs were found in our assembly (Additional file 1, Table S3).

\section{Genome annotation}

Using both de novo and library-based repetitive sequence annotation, $164.49 \mathrm{Mb}$ of repetitive elements were uncovered, accounting for $44.99 \%$ of the total assembly (Additional file 1, Table S4). The proportion of repetitive DNA in A. ramifera was similar to that in A. shenzhenica $(43.74 \%)$ but less than that in P. equestris (62\%) and $D$. catenatum (78\%). Among the repetitive sequences, transposable elements (TEs) were the most abundant $(43.1 \%)$, among which long terminal repeats (LTR) were dominant, accounting for $24.07 \%$ of the total genome (Additional file 1, Table S5 and Fig. S1).

The protein-coding gene models were predicted through a combination of de novo and homology-based annotation. In total, 22841 putative genes were identified in the A. ramifera genome, similar to that in A. shenzhenica (21 831) but less than that in V. planifolia (28 279), P. equestris (29 545), and D. catenatum (29 257) (Additional file 1, Table S6). Further functional annotation of the predicted genes was carried out by homology searches against various databases, including Gene Ontology (GO), Kyoto Encyclopedia of Genes and Genomes (KEGG), SwissProt, TrEMBL, nr database, and InterPro. Results showed that 19551 (85.6\%) predicted genes could be annotated (Additional file 1, Table S7). In addition, we identified 40 microRNA, 616 transfer RNA, 1450 ribosomal RNA, and 108 small nuclear RNA genes in the A. ramifera genome (Additional file 1, Table S8).

Synteny comparison based on gene annotations of $A$. ramifera and $A$. shenzhenica identified 927 synteny blocks with an average block size of 12.89 genes (Additional file 1, Table S9). A total of 11950 gene pairs were covered by these synteny blocks, accounting for 61 and $66 \%$ of the genome sequences of $A$. ramifera and $A$. shenzhenica, respectively (Additional file 1, Table S9). The high co-linearity between their genomes suggested a close relationship between these two species. 


\section{Gene family identification}

Gene family identification was carried out for the predicted protein-coding genes in $A$. ramifera, together with genes from other species, including $P$. equestris, $P$. aphrodite, $D$. officinale, D. catenatum, A. shenzhenica, V. planifolia, Asparagus officinalis, and Oryza sativa. A total of 19422 putative genes in the $A$. ramifera assembly were assigned to 13 251 gene families (Fig. 1 A and Additional file 1, Table S10). The remaining 3419 genes could not be grouped with other genes and were considered orphans. Among the compared species, 266 gene families were only shared by orchid species. KEGG and GO enrichment analyses of those orchid-specific gene families revealed various significantly enriched pathways and terms, including 'Stilbenoid, diarylheptanoid and gingerol biosynthesis' (ko00945), 'Zeatin biosynthesis' (ko00908), 'Flavonoid biosynthesis' (ko00941), 'Circadian rhythm - plant' (ko04712), 'Regulation of gene expression' (GO:0010468), and 'Aromatic compound biosynthetic process' (GO: 0019438) (Additional file 1, Table S11 and S12). Furthermore, a total of 1145 gene families were specifically expanded in Apostasia (see Methods), and were significantly enriched in several pathways, such as 'Ribosome biogenesis in eukaryotes' (ko03008), 'mRNA surveillance pathway' (ko03015) and 'Plant-pathogen interaction' (ko04626) (Additional file 1, Table S13 and S14).

\section{Phylogenetic analysis}

We constructed a phylogenetic tree using MrBayes with gene sequences of 381 single copy genes shared by 16 plant species, including $A$. ramifera. The divergence times among these species were estimated using PAML
MCMCTree based on our phylogeny. Results showed that the Apostasia species separated from other orchids 82 million years ago (Fig. 1B), consistent with previously published results [3]. The divergence time between $A$. ramifera and $A$. shenzhenica was estimated to be 8 million years ago (Fig. 1B). Gene family expansions and contractions on each phylogenetic branch of the 16 species were estimated using CAFE [12] (Fig. 1B). We further carried out GO/KEGG enrichment analyses on the significantly expanded gene families in A. ramifera and found some functionally enriched pathways and terms, including 'Zeatin biosynthesis' (ko00908), Glycerophospholipid metabolism (ko00564), 'Flavin adenine dinucleotide binding' (GO:0050660), and 'UDP-Nacetylmuramate dehydrogenase activity' (GO:0008762) (Additional file 1, Table S15 and S16). In addition, the significantly contracted gene families were enriched in 'Homologous recombination' (ko03440), 'Glycosphingolipid biosynthesis' (ko00604), 'Transferase activity, transferring phosphorus-containing groups' (GO:0016772), and 'Transferase activity' (GO:0016740) (Additional file 1, Table S17 and S18).

\section{History of orchid population size}

Population size history is important for understanding the underlying mechanisms leading to current patterns of species and population diversity [13]. Several investigations on orchid population size have been published $[14,15]$. Here, the pairwise sequential Markovian coalescent (PSMC) model, which uses the coalescent approach to estimate population size changes [13], was applied to infer population size history based on the genome

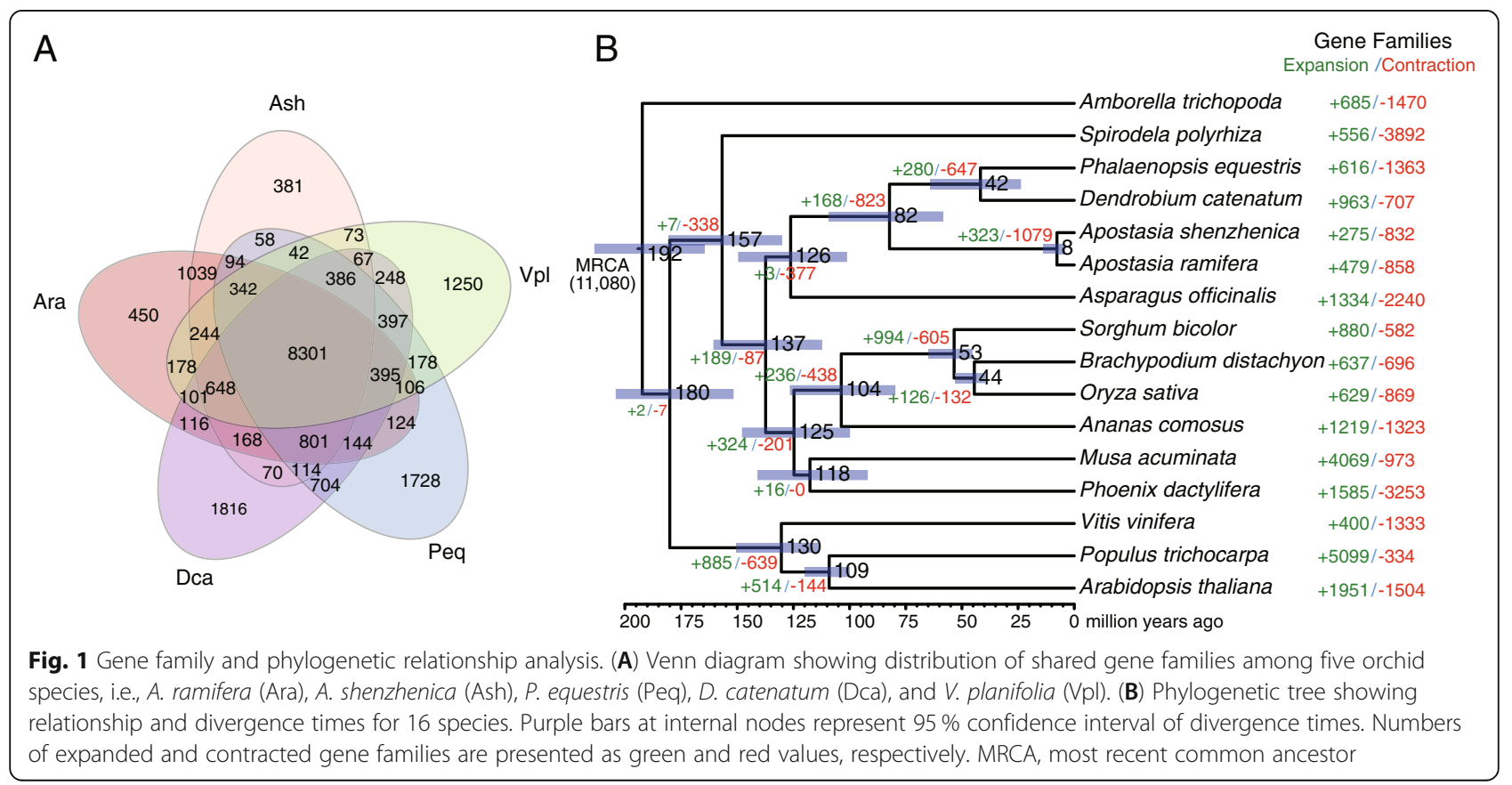


sequences of seven orchid species, i.e., A. ramifera, $A$. shenzhenica, $P$. equestris, $P$. aphrodite, $D$. officinale, $D$. catenatum, and $V$. planifolia. For the Apostasia species, population size changed between 10000 and 250000 years ago, with similar population dynamics (Fig. 2). Earlier history could not be recovered because the lowlevel heterozygosity of the genome sequences of $A$. ramifera and A. shenzhenica provided limited information on ancient changes in population size. For the other orchids, population size histories showed similar patterns, especially $D$. catenatum, $D$. officinale, and $P$. equestris (Fig. 2). First, a period of population growth was observed for each of these orchid species. Then, all orchid populations experienced a severe contraction (bottleneck) over the last 100000 years, from which they have not recovered (Fig. 2). During the reporting period (10 000 to 250000 years ago), the Apostasia species had the smallest population size compared to other orchid species. The population size of Vanilla was slightly higher than that of Apostasia, but lower than that of all Epidendroideae orchids.

\section{Gene family evolutionary analysis MADS-box transcription factors}

In plants, $M A D S$-box transcription factors are involved in various developmental processes, such as floral development, flowering control, and root growth. All MADSbox gene family members are categorized as type I or type II based on their gene tree. Using HMMER software and a $M A D S$-box domain profile (PF00319), we identified 30 putative $M A D S$-box genes in the $A$. ramifera genome, fewer than that detected in the other sequenced orchids (Additional file 1, Table S19). Phylogenetic analysis of the putative $M A D S$-box genes revealed that 23 belonged to the type II $M A D S$-box clade (Fig. $3 \mathrm{~A}$ ), fewer again than that found in other orchids, e.g., $A$. shenzhenica (27 members) [3], V. planifolia (30 members, Additional file 1, Fig. S2A), P. equestris (29) [2], and D. catenatum (35) [5]. Compared to P. equestris, there were fewer members in the $A$-class, $B$-class, $E$ class, and AGL6-class in A. ramifera and $V$. planifolia (Additional file 1, Table S19). In contrast, there were more SVP-class, ANR1-class, and AGL12-class members in $A$. ramifera and $V$. planifolia than in $P$. equestris (Additional file 1, Table S19).

Type I MADS-box transcription factors are involved in plant reproduction and endosperm development [16]. Here, we identified seven and six type I MADS-box genes in $A$. ramifera and $V$. planifolia, respectively (Fig. 3B and Additional file 1, Fig. S2B and Table S19). Phylogenetic analysis showed that genes in the $M \beta$-class were absent in $A$. ramifera and $V$. planifolia, (Fig. 3B and Additional file 1, Fig. S2B).

\section{Terpene synthase (TPS) gene family}

In plants, TPS family members are responsible for the biosynthesis of terpenoids, which are involved in various physiological processes in plants such as primary metabolism and development [17]. The architecture of the TPS gene family is proposed to be modulated by natural selection for adaptation to specific ecological niches [18]. We used both terpene_synth and terpene_synth_C domains to search for TPS genes in the orchid genomes. A small TPS gene family size was observed in the two Apostasia species compared with the other orchids studied (Fig. 4). Only eight and six copies of TPS genes were found in $A$. shenzhenica and $A$. ramifera, respectively (Fig. 4 and Additional file 1, Table S20). A small TPS family size in Apostasia may indicate a loss of chemical

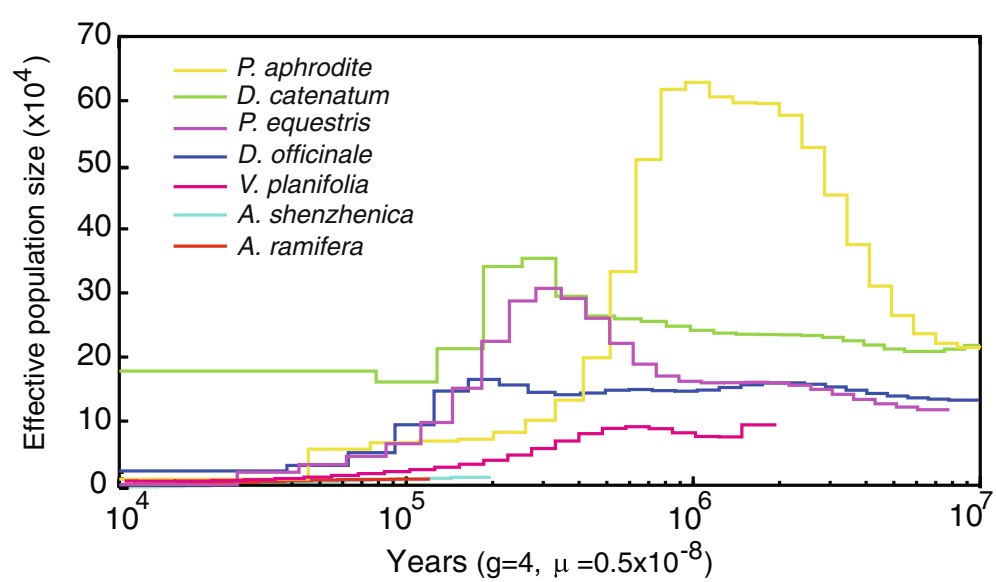

Fig. 2 Population size histories of seven orchid species, including P. aphrodite (yellow), D. catenatum (green), P. equestris (purple), D. officinale (dark blue), V. planifolia (pink), A. shenzhenica (light blue) and A. ramifera (red), between 10000 and 10 million years ago. Generation times of orchids were assumed to be four years, and mutation rate per generation was $0.5 \times 10^{-8}$ 


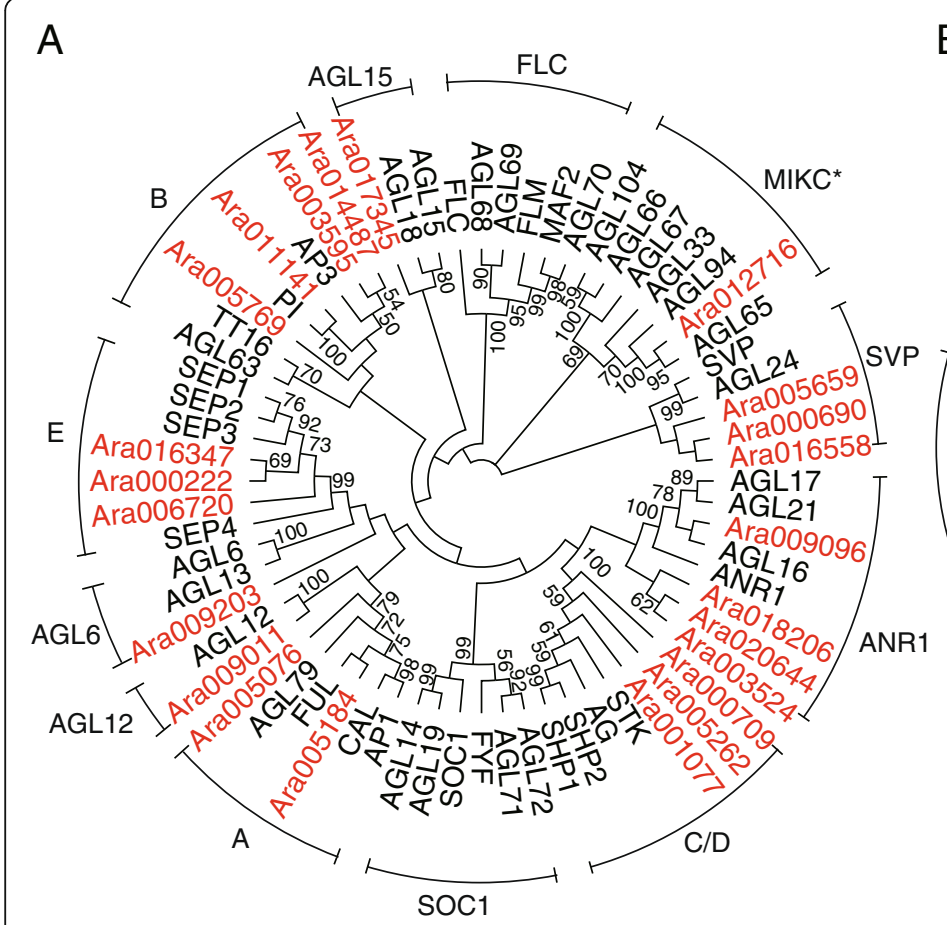

B

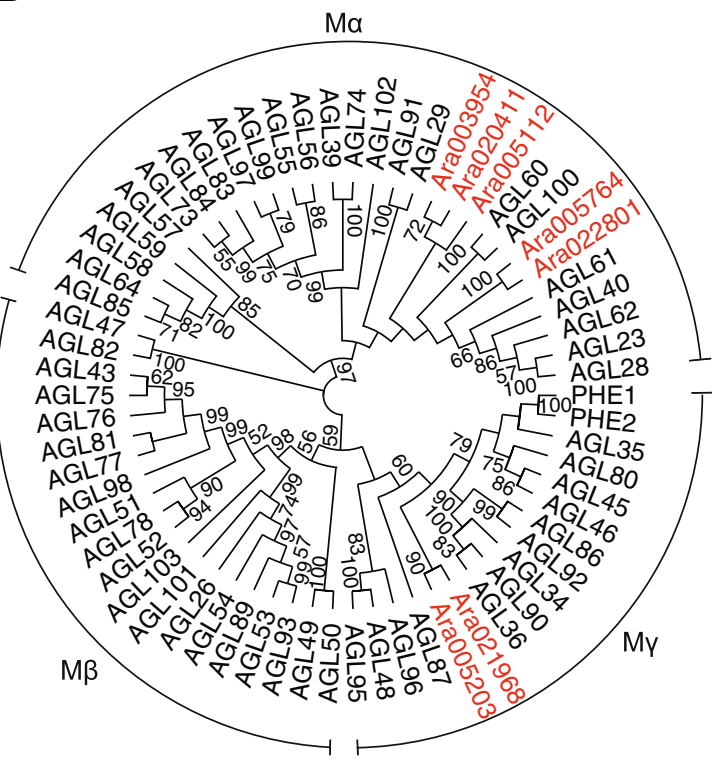

Fig. 3 Phylogenetic analysis of MADS-box genes in A. ramifera. (A) Type II MADS-box genes. (B) Type I MADS-box genes. Neighbor-joining gene trees were constructed using MADS-box genes from A. ramifera and Arabidopsis. Genes from A. ramifera are marked in red. Different MADS-box classes are indicated. Numbers above branches are bootstrap support values of at least 50

diversity of terpenoid compounds. To resolve the phylogenetic relationship of TPS genes in orchids, a gene tree was constructed using the TPS gene sequences derived from orchids and Arabidopsis. Phylogenetic analysis showed that four TPS subfamilies were found in Apostasia (Fig. 4). In Apostasia, members of both TPS-c and TPS-f subfamilies, which encode enzymes responsible for the synthesis of 20-carbon diterpenes, were lost (Fig. 4 and Additional file 1, Table S20). In addition, fewer members of TPS-a and TPS-b subfamilies were observed in Apostasia compared with other orchids (Fig. 4 and Additional file 1, Table S20). Genes from these two subfamilies are reportedly involved in the biosynthesis of 10- and 15-carbon volatile terpenoids [19], which are the components of floral scent.

\section{Pathogen resistance genes}

Pathogen resistance-related genes are closely associated with plant fitness and adaptive evolution [20]. Here, the $N B-A R C$ domain profile was used to search for $R$ genes in the predicted gene models of $A$. ramifera and other orchids, including $A$. shenzhenica, $V$. planifolia, $P$. equestris, $P$. aphrodite, $D$. catenatum, and $D$. officinale. We identified $71 R$ genes in A. ramifera and 66 in A. shenzhenica, considerably fewer than that found for $P$. equestris (114), P. aphrodite (109), D. officinale (172), D. catenatum (182), and V. planifolia (86) (Fig. 5). Thus, the size of the $R$ gene family varied greatly among the different Orchidaceae genera (Fig. 5).

In Apostasia, in addition to the small $R$ gene family size, we also discovered lower copy numbers in both the NAC and WRKY gene families (Fig. 5), which are known to play important roles in plant immune response [21, 22]. We identified 55 and 64 NAC transcription factor members in A. ramifera and A. shenzhenica, respectively, markedly fewer than that found in Dendrobium, Phalaenopsis, and Vanilla (77 to 113) (Fig. 5). We also identified 56 and 50 WRKY transcription factors in $A$. ramifera and $A$. shenzhenica, respectively, again fewer than that found in other orchids (64 to 83) (Fig. 5).

\section{Apostasia LOX1/LOX5 genes may contribute to lateral root} development, an important trait for terrestrial growth

LOX1 and LOX5 are involved in the development of lateral roots in Arabidopsis, and loss of these two genes causes a significant increase in lateral root emergence [23]. Here, we searched the homologs of LOX1 and LOX5 in six published orchid genomes using protein sequences from Arabidopsis as the query, and then constructed a gene tree to elucidate the phylogenetic relationship among these genes. We detected multiple copies of LOX1/LOX5 homologs in 


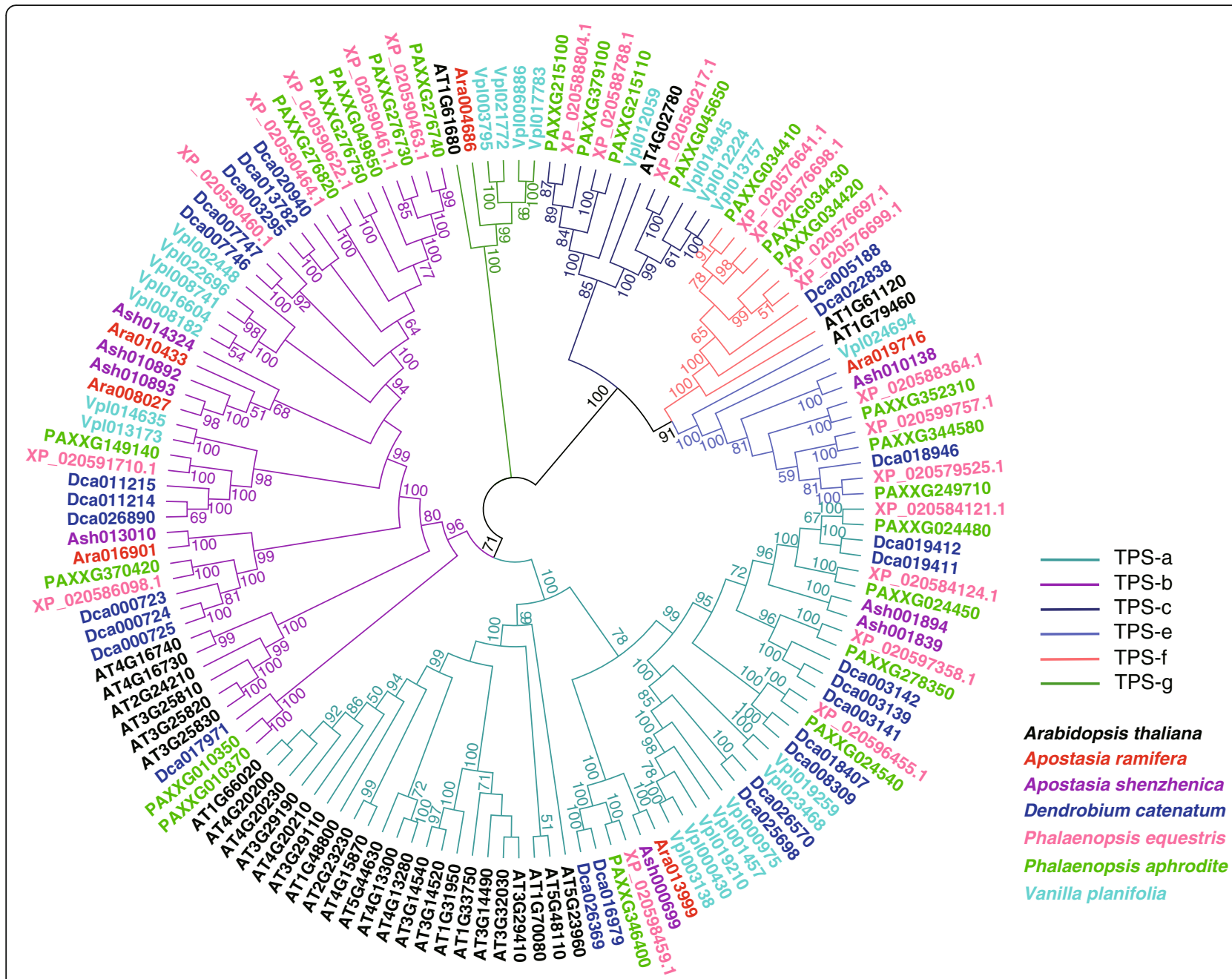

Fig. 4 Phylogenetic tree for TPS genes predicted in six orchid species and Arabidopsis. Numbers above branches are bootstrap support values of at least 50
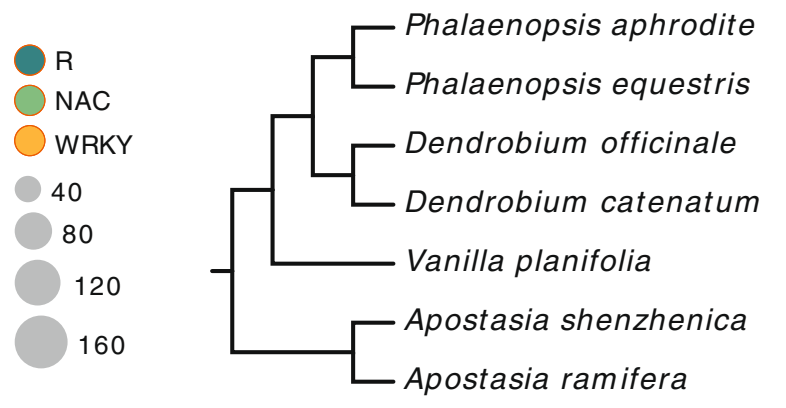

\& $\&$ \&

$109 ! 77,64$

$114 \cdot 82 \cdot 65$

$172|113| 83$

$182,91,71$

$86,92,77$

66 '64'50

71) $|55| 56$

Fig. 5 Number of members of $R$ genes and NAC and WRKY gene families in different orchids. These gene families are marked in blue, green, and yellow, respectively. Sizes of circles are directly proportional to number of members in gene family 


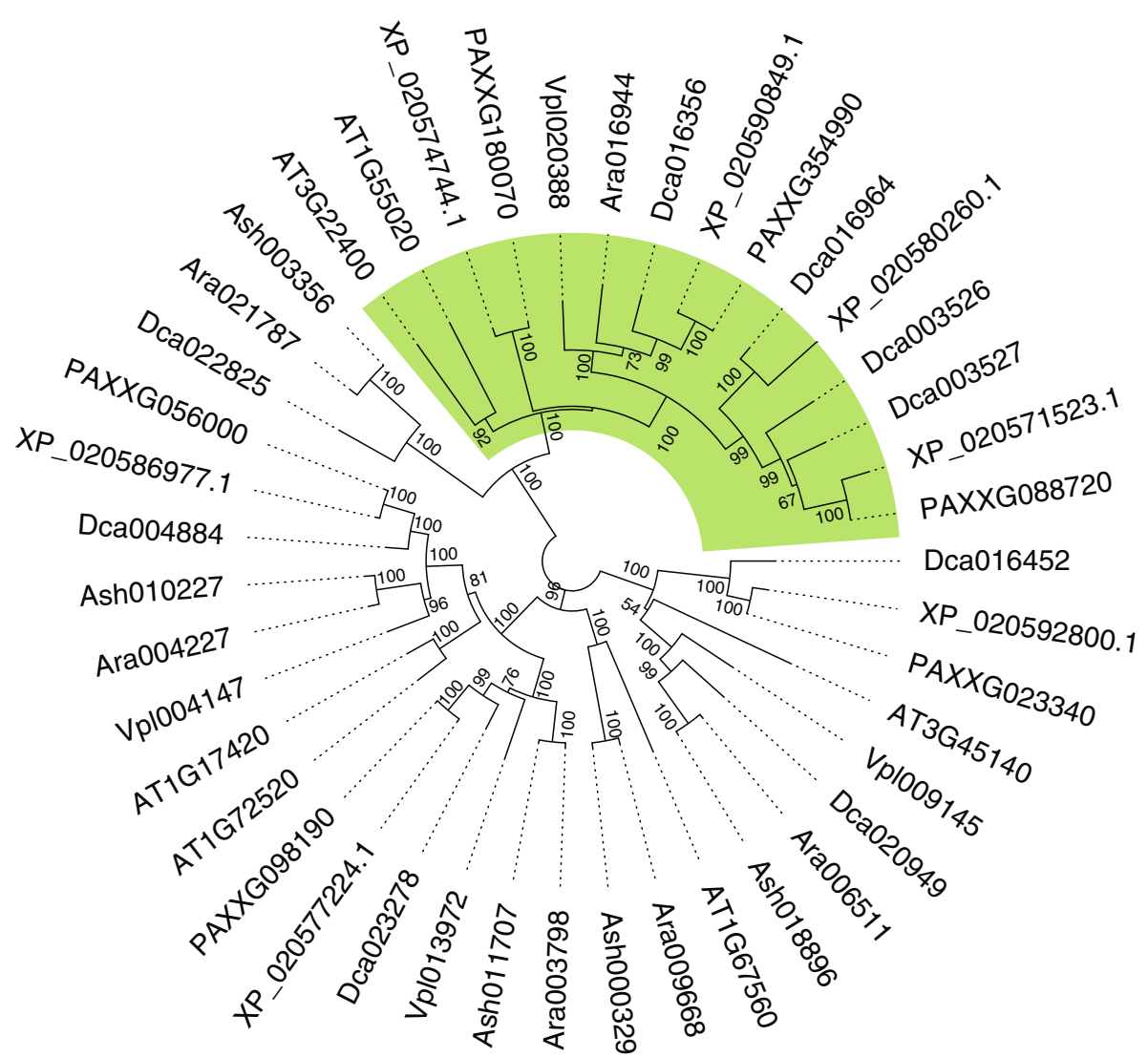

Fig. 6 LOX gene tree showing LOX1/LOX5 genes in orchids. Phylogenetic analysis was conducted using LOX gene sequences from A. ramifera, A. shenzhenica, D. catenatum, P. equestris, P. aphrodite, V. planifolia, and Arabidopsis. Branches leading to orchid LOX1/LOX5 genes are marked in green. Numbers above branches are bootstrap support values of at least 50

the epiphytic orchid genomes (Fig. 6 and Additional file 1, Table S21). However, only one homologous gene was found in A. ramifera, and the LOX1/LOX5 homologs were completely lost in $A$. shenzhenica (Fig. 6 and Additional file 1, Table S21). We also found one copy of the LOX1/LOX5 genes in the hemi-epiphytic orchid V. planifolia (Fig. 6 and Additional file 1, Table S21).

\section{Discussion}

With worldwide distribution, orchids are one of the largest flowering plant families and their extraordinary diversity provides an excellent opportunity to explore plant evolution. Certain evolutionary adaptations in orchids, e.g., pollinium, labella and epiphytism, are proposed to have played key roles in their adaptive evolution and radiation. However, the genetic basis underlying those innovations remains incompletely known. In the current study, we sequenced the genome of A. ramifera, a basal Apostasioideae lineage terrestrial orchid, and carried out comparative genomic analyses of seven orchid genomes including that of A. ramifera. Several gene families related to adaptations in orchids (e.g.,
MADS-box, pathogen resistance, TPS, and LOX genes) were compared among different orchid lineages.

\section{MADS-box transcription factors}

Compared with other orchids, we found smaller gene families in the $B$ - and $E$-classes of type II MADS genes in Apostasia and Vanilla. Genes in these classes of type II MADS are involved in floral development [24]. Furthermore, it has been proposed that small size in these gene families may be related to the maintenance of the ancestral state in Apostasia flowers, which exhibit radial symmetry and no specialized labellum [3]. However, small gene families in the $B$ - and $E$-classes of the type II MADS family were also found in $V$. planifolia, which has bilaterally symmetrical flower petals and a specialized labellum. These results indicate that members in the $B$ and $E$-classes may not contribute to the different flower morphologies found among Apostasioideae and other orchids.

Recent research has suggested that genes from the $M I K C^{*}$ family are involved in pollen development [25, 26]. Here, we found a $M I K C^{*} P$-subclass member in the $A$. ramifera genome. Furthermore, $P$ - and $S$-subclasses 
members of $M I K C^{*}$ were identified in A. shenzhenica, while $P$-subclass genes were lost in $P$. equestris [3]. It has been proposed that loss of $P$-subclass genes is associated with the evolution of pollinia [3]. However, both $P$ - and $S$-subclass members have been identified in the genome assembly of $P$. aphrodite [7] and $V$. planifolia (Additional file 1, Fig. S2). Thus, loss of $M I K C^{* *}$ genes in some orchids might not be relevant to the evolution of pollinia.

A lack of $M \beta$ genes has been reported in some orchid genomes, including A. shenzhenica, $P$. equestris, and $D$. catenatum $[2,3,5]$. Here, we found that the $M \beta$ gene was also absent in A. ramifera and $V$. planifolia. Zhang et al. [3] suggested that loss of $M \beta$-class type I MADSbox transcription factors is related to the absence of endosperm in the seeds of all orchids. However, $M \beta$ genes have been discovered in the genome of $P$. aphrodite and transcriptome of Orchis italica [7, 27]. Thus, instead of $M \beta$ genes, other genes or mechanisms may contribute to the absence of endosperm in orchid seeds.

\section{TPS gene family}

In comparison to that in Apostasia, more members in the TPS-a and TPS-b clades of the TPS gene family were found in Vanilla, Dendrobium, and Phalaenopsis. Members of these clades are involved in the biosynthesis of volatile terpenoids, which are the components of floral scent [19]. In addition, it has been proposed that expansion of TPS subfamilies may promote the emergence of novel compounds [18]. As the flowers of orchids in the Epidendroideae and Vanilloideae subfamilies are highly adapted to animal pollination via many pollination syndromes, including the development of various volatile compounds, this result may provide new insight into the genetic basis of adaptation to insect pollination in epiphytic orchids. Gene duplication and divergence are more effective ways of evolving new enzymatic functions than de novo evolution of a new gene [18]. Thus, more members of the TPS-a and TPS-b subfamilies may facilitate the emergence of novel volatile compounds, which may contribute to their adaptation to diverse animal pollinators via the production of diverse flower scents.

\section{Lateral root development}

For higher land-based plants, roots play a significant role in their successful colonization of the terrestrial environment by providing mechanical support as well as water and nutrient uptake from the soil (or air for epiphytic plants) [28]. Root architecture, i.e., the spatial organization of roots, also has a significant impact on the functional performance of the root system and is important for plant survival $[28,29]$. Environmental factors, such as water and nutrient availability, contribute to the shaping of root architecture [30]. Significant root system differences have been reported between Apostasia and other orchids [3]. Among them, branch roots have been found in Apostasia but not in epiphytic orchids, such as Phalaenopsis [3]. In land plants, the formation of lateral roots plays a crucial role in root architecture, uptake, and anchoring. Following their adaptation to soil-free environments, however, various orchids have lost the ability to develop lateral roots, instead forming specialized root structures, such as spongy epidermis, to help preserve nutrients. Zhang et al. [3] reported that variation in the copy number of ANR1 subfamily $M A D S$-box genes results in different lateral root formation between $A$. shenzhenica and epiphytic $P$. equestris and D. catenatum. However, as the development of lateral roots is a complicated process that involves intricate regulation and phytohormone interactions [31, 32], the genetic mechanisms controlling the emergence of lateral roots in orchids await further investigation. In this study, we found fewer copies of the LOX1/LOX5 homologous genes in the Apostasia species and hemi-epiphytic $V$. planifolia than that in the epiphytic orchids. Given the function of LOX1 and LOX5 in Arabidopsis [23], we propose that copy number variation in these genes may contribute to the differences in lateral root development between terrestrial and epiphytic orchids. In addition, according to the phylogenetic relationship of $L O X$ genes in orchids, there are six different subclades of $L O X$ genes in the common ancestor of orchids. The variation in copy number among the different orchid lineages may be due to the various degrees of gene retention, rather than gene duplication.

\section{Conclusions}

In this study, we performed de novo assembly and analysis of the genome of $A$. ramifera, a terrestrial orchid from the Apostasioideae subfamily. We revealed the population size histories of different orchid species and discovered a continuous decrease in population size from the genomes of these species over the last 100000 years. In addition, the gene family size and subfamily architecture of TPS genes varied greatly among species from different orchid subfamilies, which may be associated with the adaptive evolution of orchids. Genes associated with pathogen resistance were significantly reduced in the genomes of Apostasia compared with that of other orchids. In Apostasia, we also found genes that were likely involved in the regulation of lateral root development, which is an important trait for terrestrial growth. The A. ramifera genome sequence reported here should be an important resource for further investigations on orchid biology. Comparative genomics analysis of $A$. ramifera and other orchids should provide new insights into the adaptive evolution of these species. 


\section{Methods}

\section{Sample preparation and sequencing}

The $A$. ramifera samples were collected from Jianfeng Mountain, Hainan Province, China. No permission was required to collect these samples. The formal identification of plant material was conducted by Prof. Zhongjian Liu. A voucher specimen of the material was deposited at the National Orchid Conservation Center of China under deposition number Liu.JZ6475. For genome sequencing, we collected fresh leaves from A. ramifera. Extraction of genomic DNA was carried out using the modified cetyltrimethylammonium bromide protocol [33]. Five DNA libraries with different insert sizes were constructed using an Illumina library construction kit (NEB DNA Library Rapid Prep Kit for Illumina) and then sequenced using the Illumina HiSeq 2000 platform. After filtering the raw reads according to sequencing quality and adaptor contamination, a total of $57.4 \mathrm{~Gb}$ of clean data were retained for assembling the Apostasia genome.

\section{Genome assembly}

The estimated genome size of $A$. ramifera was 332.24 $\mathrm{Mb}$ according to k-mer frequency distribution. Only one peak was observed in the k-mer distribution, indicating high homozygosity of the Apostasia genome. For genome assembly, SOAPdenovo2 [34] was used for contig construction and scaffolding, and GapCloser was used for extending the length of the final contigs. In total, 57.4 Gb of clean reads derived from the DNA libraries with five insert sizes (Additional file 1, Table S1) were used by SOAPdenovo2 assembler and GapCloser for de novo genome assembly.

\section{Repeat annotation}

Repeat sequences consist of tandem repeats, such as small and micro-satellite DNA, and interspersed repeats (also known as transposable elements, TEs). In the $A$. ramifera genome, tandem repeat sequences were identified by TRF software [35]. Identification of TEs was conducted by homology searches of the RepBase database [36] and de novo prediction. Briefly, RepeatMasker [37] and RepeatProteinMask [38] were applied to identify TEs in the Apostasia genome with a RepBase-derived library of known repeat elements. For de novo prediction, we used RepeatModeler and LTR-FINDER [39] to construct a de novo repetitive element library for the $A$. ramifera genome. RepeatMasker was then applied to search the genome for TEs with the constructed database. Finally, these results were combined, and the redundant sequences were removed to generate a complete repeat annotation.

\section{Gene and non-coding RNA prediction}

Because previously published work on the $V$. planifolia draft genome [8] did not include gene prediction, we carried out protein-coding gene prediction for the A. ramifera and $V$. planifolia genomes. Firstly, we used AUGUSTUS [40] and GlimmerHMM [41] to generate the de novo predicted gene sets for our assembly and the $V$. planifolia genome (BioProject: PRJNA507095). Protein sets derived from five plant genomes, including Arabidopsis thaliana, Phalaenopsis equestris, Oryza sativa, Sorghum bicolor, and Zea mays, were then applied to search against the Apostasia and Vanilla genomes using TBLASTN with an E-value cutoff of 1e-5 and minimum query coverage of $25 \%$. GeneWise [42] was used to annotate the gene structures. The RNA-seq datasets (SRR1509356, SRR1509370, and SRR1509674) for V. planifolia were downloaded from NCBI SRA, and were de novo assembled by Trinity software. Vanilla transcripts were applied to annotate the $V$. planifolia genome using the PASA program. The annotation results derived from different methods were then integrated to generate integrated protein-coding gene sets for $A$. ramifera and $V$. planifolia with the MAKER [43] program.

Non-coding RNAs do not translate into protein sequences but exert significant roles in cellular metabolism, and include microRNAs (miRNAs), transfer RNAs (tRNAs), ribosomal RNAs (rRNAs), and small nuclear RNAs (snRNAs). Here, we applied previously described methods to search for non-coding RNAs in the Apostasia genome [3]. The miRNA- and snRNA-coding genes were predicted using INFERNAL [44] and the tRNAcoding genes were identified using tRNAscan-SE [45]. Genes encoding rRNAs were annotated by searching the genome with the rRNA sequences of Arabidopsis.

\section{Functional annotation}

Functional analysis of the predicted genes in the Apostasia genomes was performed by searching their proteincoding regions against sequences derived from publicly available databases, including Gene Ontology (GO) [46, 47], Kyoto Encyclopedia of Genes and Genomes (KEGG) [48], SwissProt [49], TrEMBL [49], non-redundant (nr) protein database, and InterProScan [50].

\section{Gene family identification}

Gene family clustering was conducted using OrthoFinder[51] with complete protein sets from seven species, including $P$. equestris, $P$. aphrodite, $D$. officinale, $D$. catenatum, $A$. shenzhenica, $A$. officinalis, and $O$. sativa, as well as the predicted protein sequences from $A$. ramifera. To limit the disturbance of alternative splicing variants on gene family clustering, the longest transcript of each gene was selected for analysis. Gene families in which the number of genes from Apostasia (including $A$. ramifera and $A$. shenzhenica) was 1.5 times 
higher than that from other orchids were considered expanded in Apostasia.

\section{Phylogenetic analysis}

To build a high-confidence phylogenetic tree, we constructed a multi-species protein set containing protein sequences from $A$. ramifera and 15 other species, including 11 monocots (Spirodela polyrhiza, D. catenatum, P. equestris, A. shenzhenica, A. officinalis, Ananas comosus, Musa acuminata, Phoenix dactylifera, Brachypodium distachyon, S. bicolor, and O. sativa), three eudicots (Vitis vinifera, A. thaliana, and Populus trichocarpa), and the outgroup Amborella trichopoda. Protein sequences that contained less than 50 amino acids were removed from the constructed dataset. The pairwise similarities between protein sequences were calculated through all-against-all BLASTP with cutoff criteria: i.e., (i) E-value $<1$ e-5, (ii) query coverage $>30 \%$, (iii) alignment identify $>30 \%$. The results were then entered into OrthoMCL [52] (v2.0.9) to construct orthologous groups. In total, 381 single-copy gene families shared by all 16 species were applied to construct a species tree using MrBayes [53] with the GTR + invgamma model. PAML MCMCTree [54] was used to estimate the species divergence times with the following time calibrations: (i) O. sativa and B. distachyon divergence time (40-54 million years ago) [55], (ii) $P$. trichocarpa and $A$. thaliana divergence time (100-120 million years ago) [56], (iii) lower boundary of monocot and eudicot divergence time (140 million years ago) [57], and (iv) upper boundary for angiosperm divergence time (200 million years ago) [58]. Gene family expansions or contractions were identified using CAFÉ [12].

\section{Heterozygosity analysis and estimation of effective population size}

Identification of heterozygous loci was performed via a previously described method [59]. Briefly, clean reads were aligned to the genome sequence of $A$. ramifera using the BWA tool [60]. Duplicate reads were then removed by Picard. SAMtools [61] was used for calling heterozygous loci, and bcftools was used for generating consensus sequences. The effective population sizes of the orchid species were estimated using the PSMC program [13]. The parameters for PSMC analysis were set to default except for $-\mathrm{g} 4$ and $-\mathrm{u} 0.5 \times 10^{-8}$.

\section{Identification of MADS-box, TPS, NAC, WRKY, R, and LOX genes}

The hidden Markov model profiles [62] were applied to search for MADS-box (Pfam Accession: PF00319), TPS (Pfam Accession: PF01397 and PF03936), NAC (Pfam Accession: PF02365), WRKY (Pfam Accession: PF03106), and $R$ (Pfam Accession: PF00931) genes using HMMER [63] (v3.2.1). $M A D S$-box genes in $A$. thaliana reported in [3] were used to reconstruct gene trees with the $M A D S$-box genes identified in A. ramifera and V. planifolia. EvolView [64] was used to visualize the number of members in the $N A C$, WRKY and $R$ gene families for the selected species. For the TPS genes, the protein sequences that possessed both Pfam domains and contained more than 500 amino acids were considered as functional genes and used for further analysis. To identify $L O X$ genes, protein sequences of the $L O X$ gene family in A. thaliana (Gene ID: AT1G55020, AT1G72520, AT1G67560, AT1G17420, AT3G22400, and AT3G45140) were used to search for homologous genes in orchids. The identified protein sequences of each gene family were aligned using MUSCLE [65] (v3.8.31) with default settings. MEGA7 [66] was then used to construct an unrooted neighborjoining tree for each gene family with 500 bootstrap replicates.

\section{Abbreviations}

TEs: Transposable elements.; GO: Gene Ontology.; KEGG: Kyoto Encyclopedia of Genes and Genomes.; PSMC: Pairwise sequential Markov coalescent.; TPS: Terpene synthase.

\section{Supplementary Information}

The online version contains supplementary material available at https://doi. org/10.1186/s12864-021-07852-3.

\section{Additional file 1.}

\section{Acknowledgements}

Not applicable.

\section{Authors' contributions}

G.Z., H.H., Z.L., and J.C. designed and managed the project. J.C. and W.Z. wrote and revised the manuscript. W.Z., G.Z., P.Z., and Y.Z. contributed to genome sequencing and data analysis. The final manuscript was read and approved by all authors.

\section{Funding}

This study was funded by the Science and Technology Development Fund Macau SAR (File no. 031/2017/A1) to H.H., Talents Team Construction Fund of Northwestern Polytechnical University (NWPU) to J.C., Fundamental Research Funds for the Central Universities (3102019JC007) to J.C., and National Thousand Youth Talents Plan to J.C. The funders played no role in the study.

\section{Availability of data and materials}

Raw data and the genome assembly from this study were deposited in NCBI under the BioProject ID: PRJNA635894. The datasets supporting the

conclusions of this article are included within the article and its additional files.

\section{Declarations}

Ethics approval and consent to participate

Not applicable.

Consent for publication

Not applicable.

\section{Competing interests}

The authors declare that they have no competing interests.

\section{Author details}

${ }^{1}$ State Key Laboratory of Quality Research in Chinese Medicine, Institute of Chinese Medical Sciences, University of Macau, 999078 Macau, China. ${ }^{2}$ Key Laboratory of National Forestry and Grassland Administration for Orchid 
Conservation and Utilization, 518114 Shenzhen, China. ${ }^{3}$ Shenzhen Key Laboratory for Orchid Conservation and Utilization, 518114 Shenzhen, China. ${ }^{4}$ National Orchid Conservation Center of China and Orchid Conservation and Research Center of Shenzhen, 518114 Shenzhen, China. ${ }^{5}$ Key Laboratory of NFGA for Orchid Conservation and Utilization, Fujian Agriculture and Forestry University, 350002 Fuzhou, China. ${ }^{6}$ School of Ecology and Environment, Northwestern Polytechnical University, 710129 Xi'an, China.

Received: 19 June 2020 Accepted: 23 June 2021

Published online: 13 July 2021

\section{References}

1. Christenhusz MJM, Byng JW. The number of known plants species in the world and its annual increase. Phytotaxa. 2016;261(3).

2. Cai J, Liu X, Vanneste K, Proost S, Tsai WC, Liu KW, et al. The genome sequence of the orchid Phalaenopsis equestris. Nat Genet. 2015;47(1):65-72.

3. Zhang GQ, Liu KW, Li Z, Lohaus R, Hsiao YY, Niu SC, et al. The Apostasia genome and the evolution of orchids. Nature. 2017;549(7672):379-83.

4. Wei S, Shih C-C, Chen N-H, Tung S-J, editors. Value chain dynamics in the Taiwan orchid industry. I International Orchid Symposium 878; 2010.

5. Zhang GQ, Xu Q, Bian C, Tsai WC, Yeh CM, Liu KW, et al. The Dendrobium catenatum Lindl. genome sequence provides insights into polysaccharide synthase, floral development and adaptive evolution. Sci Rep. 2016;6:19029.

6. Yan L, Wang X, Liu H, Tian Y, Lian J, Yang R, et al. The Genome of Dendrobium officinale Illuminates the Biology of the Important Traditional Chinese Orchid Herb. Mol Plant. 2015;8(6):922-34.

7. Chao YT, Chen WC, Chen CY, Ho HY, Yeh CH, Kuo YT, et al. Chromosomelevel assembly, genetic and physical mapping of Phalaenopsis aphrodite genome provides new insights into species adaptation and resources for orchid breeding. Plant Biotechnol J. 2018;16(12):2027-41.

8. Hu Y, Resende MF, Bombarely A, Brym M, Bassil E, Chambers AH. Genomicsbased diversity analysis of Vanilla species using a Vanilla planifolia draft genome and Genotyping-By-Sequencing. Scientific reports. 2019;9(1):1-16.

9. Kocyan A, Qiu Y-L, Endress P, Conti E. A phylogenetic analysis of Apostasioideae (Orchidaceae) based on ITS, trnL-F and matK sequences. Plant Syst Evol. 2004;247(3-4):203-13.

10. Kocyan A, Endress PK. Floral structure and development of Apostasia and Neuwiedia (Apostasioideae) and their relationships to other Orchidaceae. Int J Plant Sci. 2001;162(4):847-67.

11. Simão FA, Waterhouse RM, loannidis P, Kriventseva EV, Zdobnov EM. BUSCO: assessing genome assembly and annotation completeness with single-copy orthologs. Bioinformatics. 2015;31(19):3210-2.

12. De Bie T, Cristianini N, Demuth JP, Hahn MW. CAFE: a computational tool for the study of gene family evolution. Bioinformatics. 2006;22(10):1269-71.

13. Li $H$, Durbin $R$. Inference of human population history from individual whole-genome sequences. Nature. 2011;475(7357):493-6.

14. Gillman MP, Dodd M. The variability of orchid population size. Botanical journal of the Linnean Society. 1998;126(1-2):65-74.

15. Alexandersson R, Ågren J. Population size, pollinator visitation and fruit production in the deceptive orchid Calypso bulbosa. Oecologia. 1996;107(4): 533-40.

16. Masiero S, Colombo L, Grini PE, Schnittger A, Kater MM. The emerging importance of type I MADS box transcription factors for plant reproduction. Plant Cell. 2011;23(3):865-72.

17. Pazouki L, Niinemets Ü. Multi-substrate terpene synthases: their occurrence and physiological significance. Front Plant Sci. 2016;7:1019.

18. Karunanithi PS, Zerbe P. Terpene synthases as metabolic gatekeepers in the evolution of plant terpenoid chemical diversity. Frontiers in plant science. 2019;10:1166.

19. Chen F, Tholl D, Bohlmann J, Pichersky E. The family of terpene synthases in plants: a mid-size family of genes for specialized metabolism that is highly diversified throughout the kingdom. Plant J. 2011;66(1):212-29.

20. Tian D, Traw M, Chen J, Kreitman M, Bergelson J. Fitness costs of R-genemediated resistance in Arabidopsis thaliana. Nature. 2003;423(6935):74-7.

21. Yuan X, Wang H, Cai J, Li D, Song F. NAC transcription factors in plant immunity. Phytopathology Research. 2019;1(1):1-13.

22. Pandey SP, Somssich IE. The role of WRKY transcription factors in plant immunity. Plant physiology. 2009;150(4):1648-55.

23. Vellosillo T, Martínez M, López MA, Vicente J, Cascón T, Dolan L, et al. Oxylipins produced by the 9-lipoxygenase pathway in Arabidopsis regulate lateral root development and defense responses through a specific signaling cascade. Plant Cell. 2007;19(3):831-46.

24. Tsai W-C, Chen H-H. The orchid MADS-box genes controlling floral morphogenesis. The Scientific World Journal. 2006;6:1933-44.

25. Liu Y, Cui S, Wu F, Yan S, Lin X, Du X, et al. Functional conservation of MIKC*-Type MADS box genes in Arabidopsis and rice pollen maturation. Plant Cell. 2013;25(4):1288-303.

26. Kwantes M, Liebsch D, Verelst W. How MIKC* MADS-box genes originated and evidence for their conserved function throughout the evolution of vascular plant gametophytes. Molecular biology evolution. 2012;29(1):293-302.

27. Valoroso MC, Censullo MC, Aceto S. The MADS-box genes expressed in the inflorescence of Orchis italica (Orchidaceae). PloS one. 2019;14(3).

28. Smith S, De Smet I. Root system architecture: insights from Arabidopsis and cereal crops. The Royal Society; 2012.

29. Li X, Zeng R, Liao H. Improving crop nutrient efficiency through root architecture modifications. Journal of integrative plant biology. 2016;58(3): 193-202.

30. Kiba T, Krapp A. Plant nitrogen acquisition under low availability: regulation of uptake and root architecture. Plant Cell Physiol. 2016;57(4):707-14.

31. Du Y, Scheres B. Lateral root formation and the multiple roles of auxin. J Exp Bot. 2018;69(2):155-67.

32. Fukaki $\mathrm{H}$, Tasaka M. Hormone interactions during lateral root formation. Plant molecular biology. 2009;69(4):437.

33. Murray M, Thompson WF. Rapid isolation of high molecular weight plant DNA. Nucleic acids research. 1980;8(19):4321-6.

34. Luo R, Liu B, Xie Y, Li Z, Huang W, Yuan J, et al. SOAPdenovo2: an empirically improved memory-efficient short-read de novo assembler. Gigascience. 2012;1(1):2047-217X-1-18.

35. Benson G. Tandem repeats finder: a program to analyze DNA sequences. Nucleic acids research. 1999;27(2):573-80.

36. Jurka J, Kapitonov W, Pavlicek A, Klonowski P, Kohany O, Walichiewicz J. Repbase Update, a database of eukaryotic repetitive elements. Cytogenet Genome Res. 2005;110(1-4):462-7.

37. Tarailo-Graovac M, Chen N. Using RepeatMasker to identify repetitive elements in genomic sequences. Current protocols in bioinformatics. 2009; 25(1):4. 10. 1-4. 4.

38. Tempel S. Using and understanding RepeatMasker. Mobile Genetic Elements: Springer; 2012. pp. 29-51.

39. Xu Z, Wang H. LTR_FINDER: an efficient tool for the prediction of full-length LTR retrotransposons. Nucleic acids research. 2007;35(suppl_2):W265-W8.

40. Stanke M, Keller O, Gunduz I, Hayes A, Waack S, Morgenstern B. AUGUSTUS: ab initio prediction of alternative transcripts. Nucleic acids research. 2006; 34(suppl_2):W435-W9.

41. Majoros WH, Pertea M, Salzberg SL. TigrScan and GlimmerHMM: two open source ab initio eukaryotic gene-finders. Bioinformatics. 2004;20(16):2878-9.

42. Birney E, Clamp M, Durbin R. GeneWise and genomewise. Genome research. 2004;14(5):988-95.

43. Holt C, Yandell M. MAKER2: an annotation pipeline and genome-database management tool for second-generation genome projects. BMC Bioinform. 2011;12(1):491.

44. Nawrocki EP, Kolbe DL, Eddy SR. Infernal 1.0: inference of RNA alignments. Bioinformatics. 2009;25(10):1335-7.

45. Lowe TM, Eddy SR. tRNAscan-SE: a program for improved detection of transfer RNA genes in genomic sequence. Nucleic acids research. 1997;25(5): 955-64.

46. Ashburner M, Ball CA, Blake JA, Botstein D, Butler H, Cherry JM, et al. Gene ontology: tool for the unification of biology. Nat Genet. 2000;25(1):25-9.

47. Consortium GO. The gene ontology resource: 20 years and still GOing strong. Nucleic acids research. 2019;47(D1):D330-D8.

48. Kanehisa M, Goto S. KEGG: kyoto encyclopedia of genes and genomes. Nucleic acids research. 2000;28(1):27-30.

49. Bairoch A, Apweiler R. The SWISS-PROT protein sequence database and its supplement TrEMBL in 2000. Nucleic acids research. 2000;28(1):45-8.

50. Hunter S, Apweiler R, Attwood TK, Bairoch A, Bateman A, Binns D, et al. InterPro: the integrative protein signature database. Nucleic acids research. 2009;37(suppl_1):D211-D5.

51. Emms DM, Kelly S. OrthoFinder: solving fundamental biases in whole genome comparisons dramatically improves orthogroup inference accuracy. Genome biology. 2015;16(1):157.

52. Li L, Stoeckert CJ, Roos DS. OrthoMCL: identification of ortholog groups for eukaryotic genomes. Genome research. 2003;13(9):2178-89. 
53. Huelsenbeck JP, Ronquist F. MRBAYES: Bayesian inference of phylogenetic trees. Bioinformatics. 2001;17(8):754-5.

54. Yang Z. PAML 4: phylogenetic analysis by maximum likelihood. Molecular biology evolution. 2007;24(8):1586-91.

55. Initiative IB. Genome sequencing and analysis of the model grass Brachypodium distachyon. Nature. 2010;463(7282):763.

56. Tuskan GA, Difazio S, Jansson S, Bohlmann J, Grigoriev I, Hellsten U, et al. The genome of black cottonwood, Populus trichocarpa (Torr. \& Gray). science. 2006;313(5793):1596-604.

57. Chaw S-M, Chang C-C, Chen H-L, Li W-H. Dating the monocot-dicot divergence and the origin of core eudicots using whole chloroplast genomes. Journal of molecular evolution. 2004;58(4):424-41.

58. Magallón S, Hilu KW, Quandt D. Land plant evolutionary timeline: gene effects are secondary to fossil constraints in relaxed clock estimation of age and substitution rates. Am J Bot. 2013:100(3):556-73.

59. Chaw S-M, Liu Y-C, Wu Y-W, Wang H-Y, Lin C-YI, Wu C-S, et al. Stout camphor tree genome fills gaps in understanding of flowering plant genome evolution. Nature plants. 2019;5(1):63-73.

60. Li H, Durbin R. Fast and accurate short read alignment with BurrowsWheeler transform. bioinformatics. 2009;25(14):1754-60.

61. Li H, Handsaker B, Wysoker A, Fennell T, Ruan J, Homer N, et al. The sequence alignment/map format and SAMtools. Bioinformatics. 2009;25(16): 2078-9.

62. El-Gebali S, Mistry J, Bateman A, Eddy SR, Luciani A, Potter SC, et al. The Pfam protein families database in 2019. Nucleic acids research. 2019;47(D1): D427-D32

63. Eddy SR. Accelerated profile HMM searches. PLoS computational biology. 2011;7(10).

64. Subramanian B, Gao S, Lercher MJ, Hu S, Chen W-H. Evolview v3: a webserver for visualization, annotation, and management of phylogenetic trees. Nucleic acids research. 2019:47(W1):W270-W5.

65. Edgar RC. MUSCLE: multiple sequence alignment with high accuracy and high throughput. Nucleic acids research. 2004;32(5):1792-7.

66. Kumar S, Stecher G, Tamura K. MEGA7: molecular evolutionary genetics analysis version 7.0 for bigger datasets. Molecular biology evolution. 2016; 33(7):1870-4.

\section{Publisher's Note}

Springer Nature remains neutral with regard to jurisdictional claims in published maps and institutional affiliations.

Ready to submit your research? Choose BMC and benefit from:

- fast, convenient online submission

- thorough peer review by experienced researchers in your field

- rapid publication on acceptance

- support for research data, including large and complex data types

- gold Open Access which fosters wider collaboration and increased citations

- maximum visibility for your research: over $100 \mathrm{M}$ website views per year

At BMC, research is always in progress.

Learn more biomedcentral.com/submissions 